\title{
Relationship of Secreted Salivary Protein Variants to Virulence in Hessian Fly (Mayetiola destructor (Say))
}

\author{
Yan M. Crane ${ }^{1,2}$ (), Charles F. Crane ${ }^{1,3}$ (), Brandon J. Schemerhorn ${ }^{1,2 *}$ (1) \\ ${ }^{1}$ USDA-ARS Crop Production and Pest Control Research Unit, West Lafayette, IN, USA \\ ${ }^{2}$ Department of Entomology, Purdue University, West Lafayette, IN, USA \\ ${ }^{3}$ Department of Botany and Plant Pathology, Purdue University, West Lafayette, IN, USA \\ Email: *brandi.schemerhorn@usda.gov
}

How to cite this paper: Crane, Y.M., Crane, C.F. and Schemerhorn, B.J. (2020) Relationship of Secreted Salivary Protein Variants to Virulence in Hessian Fly (Mayetiola destructor(Say)). Advances in Entomology, 8, 15-33.

https://doi.org/10.4236/ae.2020.81002

Received: September 28, 2019

Accepted: November 2, 2019

Published: November 5, 2019

Copyright $\odot 2020$ by author(s) and Scientific Research Publishing Inc. This work is licensed under the Creative Commons Attribution International License (CC BY 4.0).

http://creativecommons.org/licenses/by/4.0/

\begin{abstract}
Salivary proteins are the initial contact between sedentary insect pests and their host plants. It is expected that one or more salivary proteins mediate the interaction between Hessian fly and wheat, in which a feeding site is established to the benefit of the fly. A survey of 52 loci annotated as insect secreted salivary proteins was conducted in 384 individuals evenly distributed among eight biotypes of Hessian fly (B, C, D, E, GP, L, O, and vH9). Amplicons were sequenced with Illumina, and sequence reads were aligned to the reference sequences from which primers had been designed. Positions of consistent base variation (998 in all) were identified and tabulated by biotype. No varying position was associated with biotype-wide virulence to any one of wheat resistance genes $\mathrm{H} 3, \mathrm{H} 5, \mathrm{H} 6, \mathrm{H} 7 / \mathrm{H} 8, \mathrm{H} 9, \mathrm{H} 11, \mathrm{H} 13$, and $\mathrm{H} 26$. The multiplate pooling strategy utilized in this study is an effective, affordable way to reveal the genotype of hundreds of individuals at tens of genetic loci.
\end{abstract}

\section{Keywords}

Hessian Fly, Mayetiola destructor, Virulence, Insect-Plant Interaction, Amplicon Sequencing

\section{Introduction}

As the third largest producer of wheat in the world, the United States exported 27.2 million tonnes of wheat during the 2018-19 growing season [1]. To fight wheat yield loss due to Hessian fly infestation, it is necessary to be able to identify the molecular mechanisms that operate during wheat-Hessian fly interac- 
tions. Secreted salivary proteins are the major point of contact between sucking insects and their plant hosts. There is considerable evidence that some secreted salivary proteins trigger defense responses, while others suppress ongoing defense responses [2]. Virulence requires that the host response is sufficiently suppressed to allow the insect to survive and reproduce. In Hessian fly (Mayetiola destructor (Say)), eight biotypes have been defined by their pattern of virulence against a battery of wheat host resistance genes [3] [4] [5]. Here we report an investigation of the distribution of polymorphic nucleotides for 52 secreted salivary proteins in samples of eight biotypes that had been maintained for many years in laboratory culture, since these sites would be potential biomarkers for virulence. We looked for correlation between specific polymorphic sites and biotype, i.e., virulence to a particular set of wheat $\mathrm{R}$ genes.

\section{Materials and Methods}

\subsection{Source of Sequence}

Translated dipteran nucleotide sequence in GenBank nt was screened for signal peptides with SignalP [6], and 52 such sequences (Supplementary Table 1) were chosen for primer construction and amplification. Each $20 \mu \mathrm{l}$ PCR was performed by using $10 \mu \mathrm{l}$ MyFi Mix from Bioline (Taunton, MA USA), $1 \mu \mathrm{l}$ of sample DNA in water, and $1 \mu \mathrm{l}$ of each primer for a final primer concentration of $0.5 \mu \mathrm{M}$. Initial denaturation was at $95^{\circ} \mathrm{C}$ for $2 \mathrm{~min}$ followed by 35 cycles of denaturation at $95^{\circ} \mathrm{C}$ for $30 \mathrm{sec}$, annealing at $55^{\circ} \mathrm{C}$ for $30 \mathrm{sec}$, and extension at $72^{\circ} \mathrm{C}$ for $1 \mathrm{~min}$. The PCR finished with extension at $72^{\circ} \mathrm{C}$ for $5 \mathrm{~min}$ and holding at $4^{\circ} \mathrm{C}$. A multi-plate multiplexing strategy was applied to minimize sequencing cost (Figure 1). The four 96-well plates were sequenced as $2 \times 250$ base-pair paired-end Illumina reads in samples of 48 individuals from each of Hessian fly biotypes B, C, D, E, GP, L, O and vH9 [7].

\subsection{SNP or Indel Discovery}

The PCR multiplexing strategy appears as the list of barcodes in Figure 1. Reads

Table 1 . Hessian fly biotype virulence to wheat $\mathrm{R}$ genes.

\begin{tabular}{ccccccccc}
\hline & H3 & H5 & H6 & H7/8 & H9 & H11 & H13 & H26 \\
\hline B & + & - & - & + & - & - & - & + \\
C & - & - & + & + & + & - & - & + \\
D & + & - & + & + & - & - & - & + \\
E & + & - & - & - & - & - & + & + \\
GP & - & - & - & - & - & - & - & - \\
L & + & + & + & + & - & MS & - & - \\
O & + & + & + & - & - & + & - & + \\
vH9 & + & MS & + & + & + & MS & - & MS \\
\hline
\end{tabular}

+virulent, $>90 \%$ survival on wheat carrying this R gene; -avirulent, $<10 \%$ survival on wheat carrying this $\mathrm{R}$ gene; MS, missing datum, i.e., ambiguous virulence consistent with individual polymorphism. 


\section{$1^{\text {st }}$ PCR}

Adapterf(20bp): CTAAATGTTACCGCCGTGAG

Template-specific-forward primer
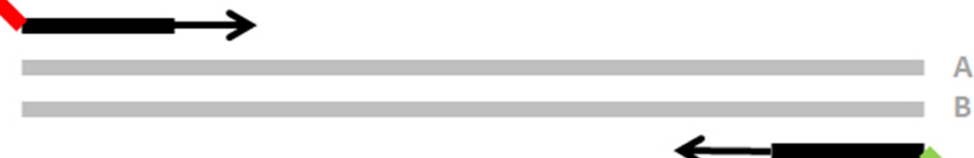

Template-specific-reverse primer

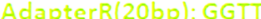

$2^{\text {nd }}$ PCR

TruSeq Front End: CAAGCAGAAGACGgCATACGAGAT

Column Barcode (8bp each ${ }^{2}$ )

TruSeq Back End: GTGACTGGAGTTCAGACGTGTGCTCTTCCGATCT

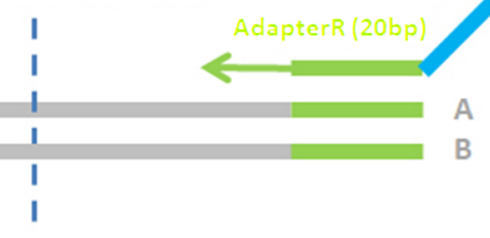

AdapterF (20bp)

TruSeq Back End: ACACTCTTTCCCTACACGACGCTCTTCCGATCT

Row Barcode (8bp each ${ }^{1}$ )

TruSeq Front End: AATGATACGGCGACCACCGAGATCTACAC

1 rA: TGAACCT, rB: TGCTAAGT, rC: TGTTCTCT, rD: TAAGACAC, rE: CTAATCGA, rF: CTAGAACA, rG: TAAGTTCC, rH: TAGACCTA

2 c1: ATCACGAC, C2: ACAGTGGT, c3: CAGATCCA, C4: ACAAACGG, C5: ACCCAGCA, C6: AACCCCTC, c7: CCCAACCT, C8: CACCACAC, C9: GAAACCCA, C10: TGTGACCA, C11: AGGGTCAA, C12: AGGAGTGG

Figure 1. Amplicon construction for the 52 loci.

were grouped by primer pair, and each group was aligned and displayed in the Integrative Genomics Viewer [8]. Single nucleotide variants (SNPs) were detected by visual inspection. Coordinates of each combination of locus (primer pair) and variable site were saved to a file for each biotype from the Integrative Genomics Viewer.

\subsection{Tabulation and Display of Variable Sites}

The number of variable sites shared by each possible combination of biotypes was calculated from the coordinates files using the Bioinformatics and Evolutionary Genomics website of the Vib-Ugent Center for Plant Systems Biology of Ghent University [9]. Combinations were enumerated three ways, for all eight biotypes B through vH9 listed above, for the seven biotypes without vH9, and for the six biotypes without $\mathrm{vH} 9$ and $\mathrm{L}$, to investigate the sharing of variable sites among biotypes to the exclusion of other biotypes (Table 1). The combination counts were presented as partial or complete Venn diagrams of greatly differing appearance, because the problem of drawing such high-order Venn diagrams has not yet been solved completely. The eight-biotype diagram only showed the numbers of variable sites within each biotype and shared in 12 of 28 possible pairs of biotypes (Figure 2); it was produced using the Venn Diagram Maker Online [10], and the size of each overlap in the diagram reflected the number of shared variable sites. The complete seven-biotype diagram (Figure 3) was drawn in the Adelaide format using R package venn [11]. The complete six-biotype di- 
agram (Figure 4) was drawn with tools in the same Vib-Ugent website [9] that counted the combinations.

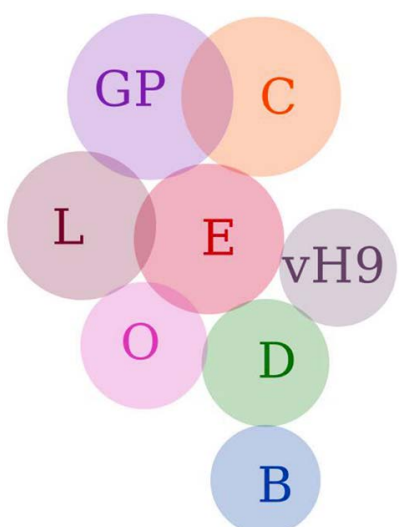

Figure 2. Partial Venn diagram of the number of variable sites in common in pairs of biotypes.

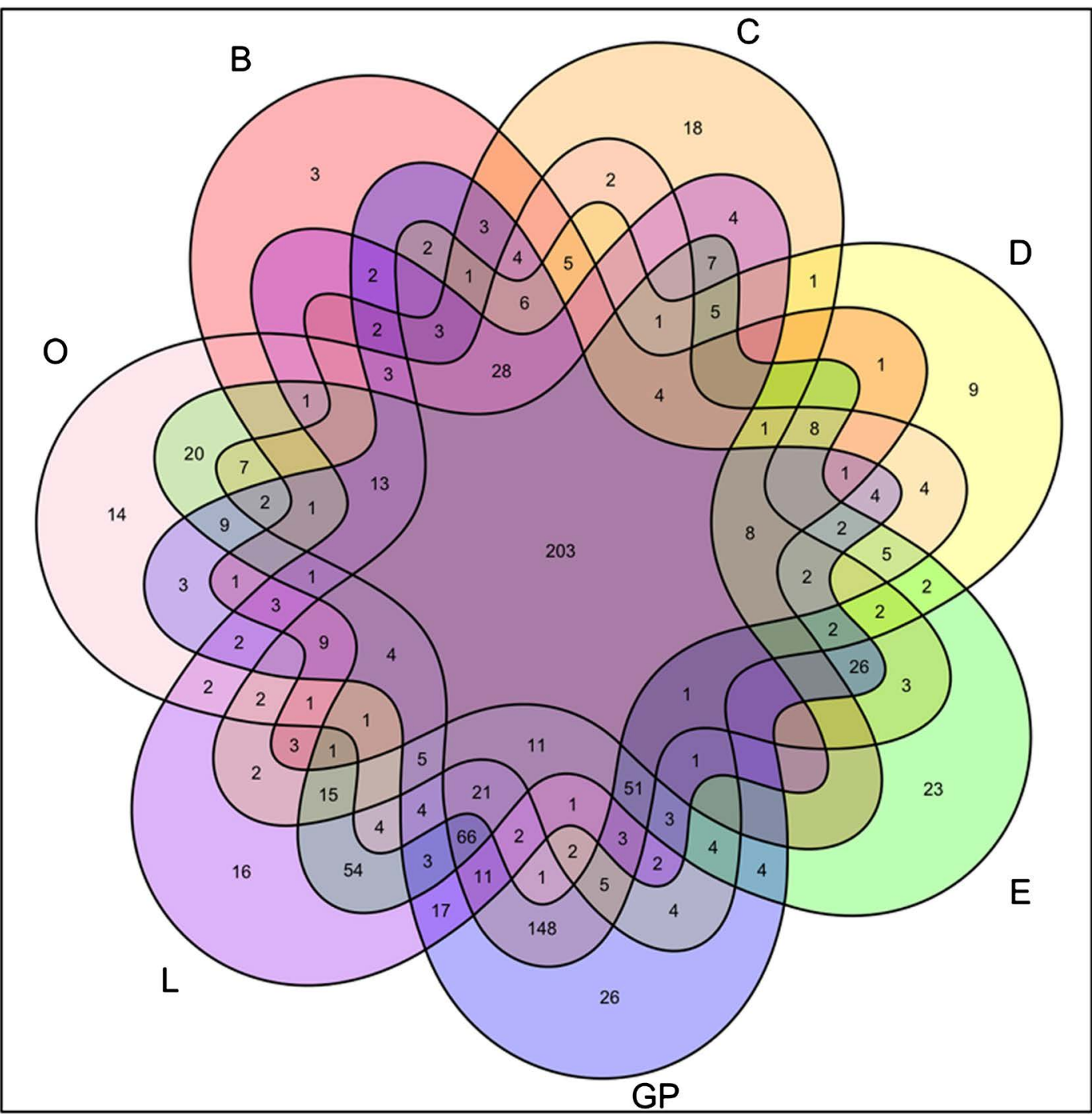

Figure 3. Numbers of variable sites in common in the biotype combinations that exclude vH9, because the drawing software cannot display a Venn diagram of eight entities with all possible intersections. 


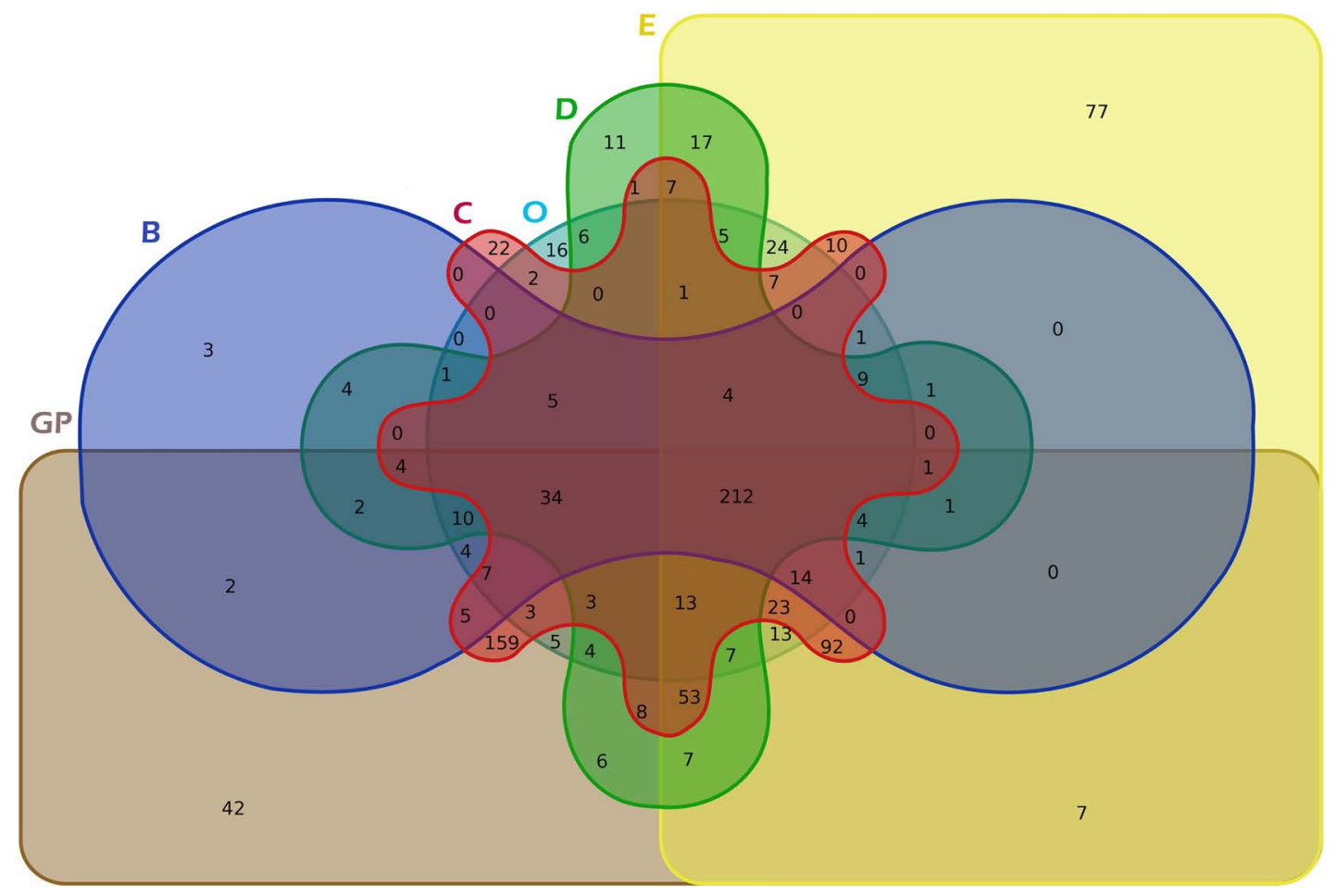

Figure 4. Numbers of variable sites in common in all biotype combinations that exclude L and vH9.

\section{Results and Discussion}

Illumina sequencing produced 27,247,176 merged read pairs that passed quality control and were greater than 150 bases in length. The counts of read pairs ranged from 79,684 for S42 to 1,846,094 for S31 and from 1,148,582 for biotype C to 2,808,266 for biotype D. Counts of read pairs ranged from 261 to 123,110 for individual flies over all loci.

The biotypes display distinct combinations of virulence and avirulence to alleles at eight wheat $R$ genes as shown in Table 1 [12]. Thus biotypes $B, D, E, L$, $\mathrm{O}$, and $\mathrm{vH} 9$ all survive on $\mathrm{H} 3$ wheat, but they share no polymorphic sites in our set of 52 secreted salivary proteins to the exclusion of C and GP (Table 2). Likewise biotypes $\mathrm{C}, \mathrm{D}, \mathrm{L}, \mathrm{O}$, and $\mathrm{vH} 9$ overcome $\mathrm{H} 6$ but share no polymorphic sites to the exclusion of B, E, and GP; B, C, D, L, and vH9 overcome $\mathrm{H} 7 / \mathrm{H} 8$ and share no polymorphic sites to the exclusion of $\mathrm{E}, \mathrm{GP}$, and $\mathrm{O}$; and $\mathrm{C}$ and $\mathrm{vH} 9$ overcome H9 but share no polymorphic sites to the exclusion of B, D, E, GP, L, and O (Table 2). The relative sizes of circles and overlaps in Figure 2 show the sharing or lack thereof of 105 positions for single biotypes and 256 positions for pairs of biotypes, but it does not show the 637 polymorphic positions shared among three or more biotypes. The pairs C-GP (148 exclusively shared sites, Table 2), E-L (39 sites), E-O (20 sites), and GP-L (16 sites) have the most sharing to the exclusion of other relationships.

Among the tested biotypes, only E overcomes H13 (Table 2). It uniquely harbors 22 variable positions, but 13 sites of substitution and one site of deletion reside in one acetylcholinesterase locus, which matches GenBank accession 
Table 2. Numbers of variable sites in common only among given combinations of eight biotypes.

\begin{tabular}{|c|c|c|c|c|c|c|c|c|c|c|c|c|c|c|c|c|}
\hline & & $\mathrm{Gp}$ & $\mathrm{L}$ & $\mathrm{O}$ & vH9 & GpL & $\mathrm{GpO}$ & GpvH9 & LO & LvH9 & OvH9 & GpLO & GpLvH9 & GpOvH9 & LOvH9 & GpLOvH9 \\
\hline & & 25 & 15 & 13 & 2 & 16 & 3 & 0 & 2 & 1 & 1 & 1 & 1 & 0 & 0 & 1 \\
\hline B & 3 & 0 & 0 & 0 & 0 & 2 & 1 & 0 & 0 & 0 & 0 & 3 & 0 & 0 & 0 & 0 \\
\hline C & 18 & 148 & 2 & 1 & 0 & 10 & 0 & 0 & 0 & 2 & 1 & 2 & 1 & 1 & 0 & 0 \\
\hline $\mathrm{D}$ & 7 & 4 & 1 & 4 & 2 & 1 & 3 & 0 & 1 & 1 & 0 & 0 & 1 & 1 & 1 & 0 \\
\hline E & 22 & 4 & 39 & 20 & 1 & 3 & 9 & 0 & 3 & 15 & 0 & 4 & 0 & 0 & 1 & 0 \\
\hline $\mathrm{BC}$ & 0 & 3 & 0 & 0 & 0 & 2 & 4 & 0 & 0 & 0 & 0 & 3 & 0 & 0 & 0 & 0 \\
\hline $\mathrm{BD}$ & 1 & 2 & 3 & 0 & 0 & 0 & 1 & 0 & 1 & 0 & 0 & 5 & 0 & 0 & 0 & 4 \\
\hline $\mathrm{BE}$ & 0 & 0 & 0 & 0 & 0 & 0 & 0 & 0 & 1 & 0 & 0 & 0 & 0 & 0 & 0 & 1 \\
\hline $\mathrm{CD}$ & 1 & 4 & 0 & 0 & 0 & 1 & 1 & 1 & 0 & 0 & 0 & 0 & 2 & 1 & 0 & 1 \\
\hline $\mathrm{CE}$ & 3 & 20 & 5 & 6 & 0 & 43 & 2 & 6 & 0 & 2 & 1 & 21 & 23 & 0 & 0 & 0 \\
\hline $\mathrm{DE}$ & 2 & 4 & 2 & 4 & 0 & 3 & 0 & 0 & 0 & 13 & 1 & 2 & 0 & 2 & 0 & 3 \\
\hline $\mathrm{BCD}$ & 0 & 0 & 0 & 5 & 0 & 0 & 4 & 1 & 0 & 0 & 0 & 19 & 3 & 2 & 0 & 9 \\
\hline $\mathrm{BCE}$ & 0 & 0 & 0 & 0 & 0 & 0 & 1 & 0 & 0 & 0 & 0 & 6 & 0 & 0 & 0 & 7 \\
\hline $\mathrm{BDE}$ & 0 & 0 & 1 & 1 & 0 & 1 & 0 & 0 & 1 & 0 & 7 & 2 & 0 & 0 & 0 & 2 \\
\hline $\mathrm{CDE}$ & 2 & 1 & 1 & 0 & 0 & 10 & 2 & 1 & 1 & 4 & 0 & 4 & 41 & 0 & 0 & 7 \\
\hline BCDE & 0 & 0 & 0 & 1 & 0 & 1 & 2 & 0 & 3 & 0 & 0 & 15 & 0 & 6 & 0 & 189 \\
\hline
\end{tabular}

For example, biotypes C, E, GP, and L have 43 variant sites in common among the 52 amplicons, and variability at these sites is not shared with any other biotype.

ATY49611.1. However, the vH13 gene has previously been mapped and characterized (GenBank accession AEG42082.1) [13], so the distinct variant positions in the acetylcholinesterase of biotype E might be merely coincidental.

Biotypes $\mathrm{L}$ and $\mathrm{O}$ overcome $\mathrm{H} 5$, but the response of $\mathrm{vH} 9$ to $\mathrm{H} 5$ is not known with certainty. Biotypes $\mathrm{L}$ and $\mathrm{O}$ uniquely share two variable sites, and the trio of $\mathrm{L}, \mathrm{O}$, and vH9 shares no variable sites (Table 2). The variants in $\mathrm{L}$ and $\mathrm{O}$ occur at base 40 in locus 78 (a peroxidase; Figure 5(a)) and base 236 in locus 144 (lysosomal acid phosphatase; Figure 5(b)), but both occur in only $2 \%-5 \%$ of individuals and therefore cannot account for the overall virulence of $\mathrm{L}$ and $\mathrm{O}$ toward H5. Biotypes B, C, D, E, and O overcome H26. These biotypes share polymorphism at base 120 in S34, a sequence similar to Genbank accession XP_020706750.1 (endothelin-converting enzyme; Figure 5(c)), again at too low frequency to account for the virulence of these biotypes at large to H26. Only biotype O overcomes $\mathrm{H} 11$, but the response of $\mathrm{L}$ and $\mathrm{vH} 9$ to $\mathrm{H} 11$ is not known with certainty; O has 16 apparently unique polymorphic sites.

Figure 3 and Figure 4 show the distributions of uniquely shared polymorphic sites when $\mathrm{vH} 9$ or $\mathrm{vH} 9$ and $\mathrm{L}$ are not considered. The counts differ somewhat from those in Table 2, where all eight biotypes are represented. All three depictions show a large core of 189 to 212 locus-positions that vary in all the represented biotypes and therefore do not appear to be directly involved in virulence. Also, biotype GP uniquely shares more variable positions than any other 


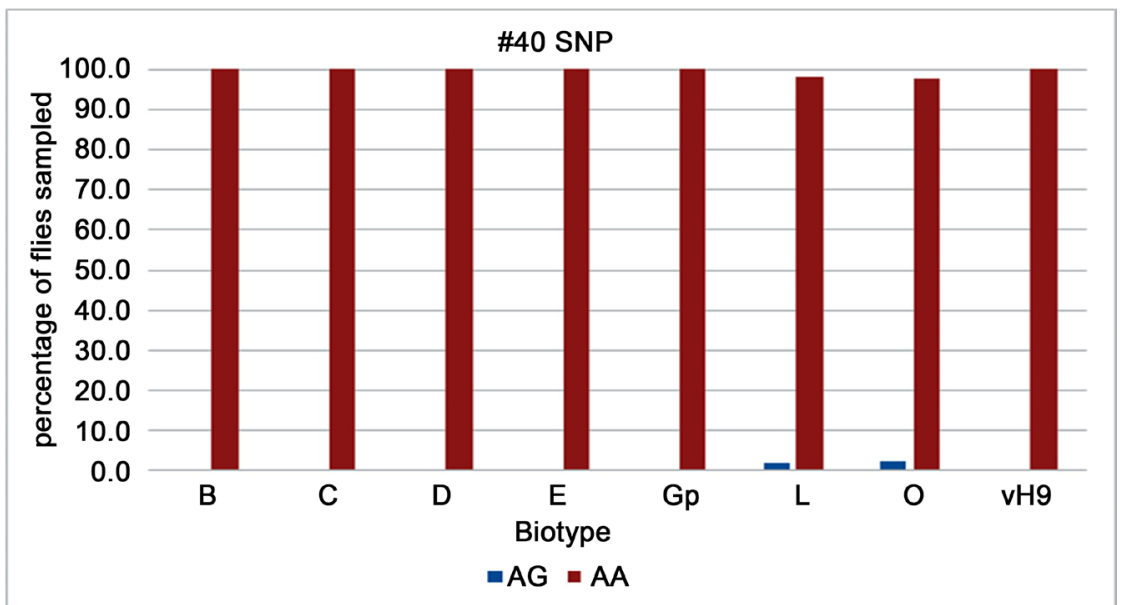

(a)

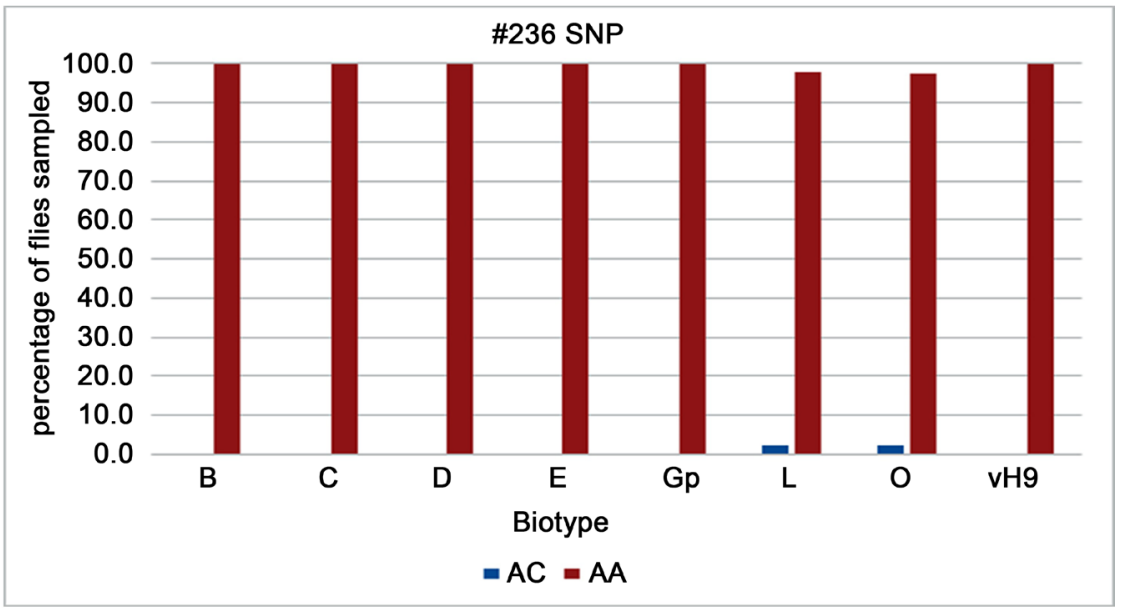

(b)

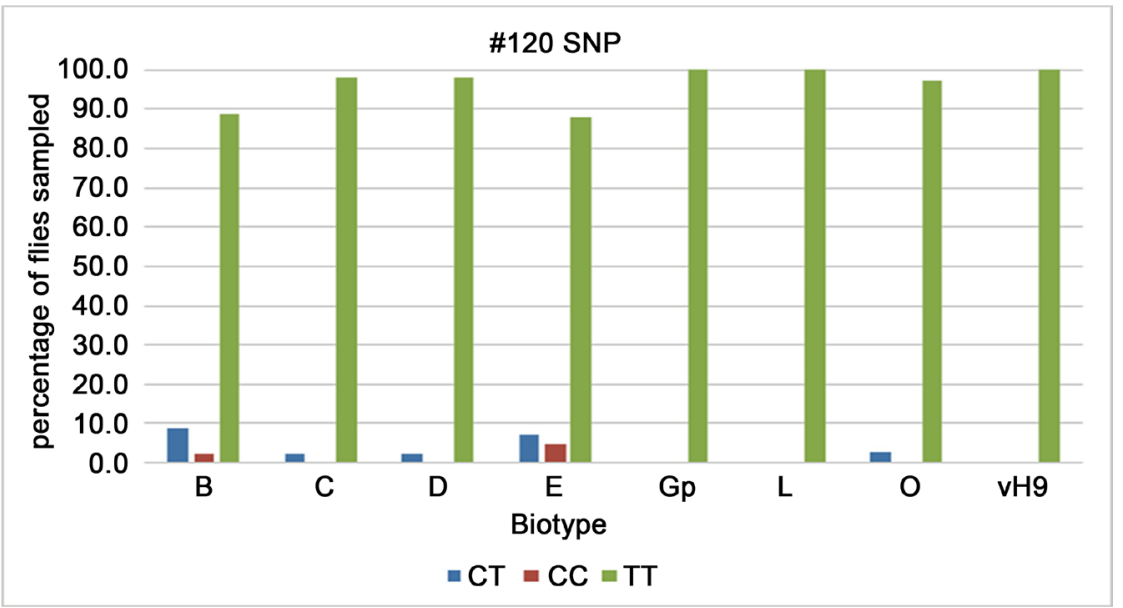

(c)

Figure 5. Within-biotype genotype frequencies in particular loci that are polymorphic in biotypes virulent to $\mathrm{H} 5$ and H26. (a) Genotype frequencies by biotype at nucleotide 40 in locus S78, which matches GenBank ETN66032.1, peroxidase from Anopheles darlin$g i$; (b) Genotype frequencies by biotype at nucleotide 236 in locus S144, which matches GenBank KYN33573.1, lysosomal acid phosphatase 1, partial, from Drosophila nasuta; (c) Genotype frequencies at nucleotide 120 in locus S34, which matches GenBank XP_020706750.1, endothelin-converting enzyme homolog isoform X1 from Athalia rosae. 
biotype; $\mathrm{L}$ is a distant second. Biotype GP is not virulent against any of the listed resistance genes [14] and thus might be the least modified from ancestral populations existing before the deployment of wheat $\mathrm{R}$ genes.

A multiplate pooling strategy was cost-effective and yet allowed reads to be traced to individual fly. This strategy was successfully applied to a panel of 52 genes that encode signal peptides and whose protein products are expectedly secreted. Nevertheless, no frequent nucleotide variant at any particular polymorphic site was limited to biotypes that shared a common virulence toward any of eight wheat resistance genes, and thus none of the 52 queried genes seems to cause virulence.

A more sensitive method to detect gene involvement would be to look at alleles as whole-amplicon variants, but even this is not free of errors. One attempt was to 1) map all the reads to one of the 52 genes, 2) assemble those reads that mapped to any one locus with SPAdes [15], 3) determine individual fly genotype by blastn [16] of the reads against the assembly, and 4) look for high-frequency variant alleles shared by biotypes virulent to a particular resistance gene. This attempt was complicated by the large number of spurious "alleles" arising from assembled sequencing errors, such that the actual alleles comprised less than $5 \%$ of the total assembly and accounted for less than $70 \%$ of the read counts. Nevertheless, once optimized this method might (or might not) reveal a relationship of a particular multiSNP allele to virulence to a particular resistance gene. A more robust method is linkage mapping with markers traceable to regions in the Hessian fly genome assembly, as performed by Zhao et al. [17] with microsatellites to identify $\mathrm{vH} 24$.

\section{Acknowledgements}

This work was supported by USDA-ARS Research Project Plan 5020-22000-01800D. We thank Sue Cambron and Jill Nemacheck for helpful advice regarding the virulence profile of biotypes and Lucy Springmeyer for assisting with organizing the data from this work. Mention of a commercial or proprietary product does not constitute an endorsement by the U.S. Federal Government.

\section{Conflicts of Interest}

The authors declare no conflicts of interest regarding the publication of this paper.

\section{References}

[1] Statista (2019) U.S. Imports and Exports of Wheat from 2000/01 to 2018/19 (in Million Metric Tons). https://www.statista.com/statistics/237902/us-wheat-imports-and-exports-since-200 $\underline{0}$

[2] Chaudhary, R., Peng, H.-C., He, J., MacWilliams, J., Teixeira, M., Tsuchiya, T., Chesnais, Q., Mudgett, M.B. and Kaloshian, I. (2018) Aphid Effector Me10 Interacts with Tomato TFT7, a 14-3-3 Isoform Involved in Aphid Resistance. New Phytolo- 
gist, 221, 1518-1528. https://doi.org/10.1111/nph.15475

[3] Cartwright, W.B. and Noble, W.B. (1947) Studies on Biological Races of the Hessian Fly. Journal of Agricultural Research, 75, 147-153.

[4] Kudagamage, C., Foster, J.E., Taylor, P.L. and Chen, B.H. (1990) Biotypes of the Hessian Fly (Diptera: Cecidomyiidae) Identified in the Southeastern United States. Journal of Entomological Science, 25, 575-580. https://doi.org/10.18474/0749-8004-25.4.575

[5] Ratcliffe, R.H., Safranski, G.G., Patterson, F.L., Ohm, H.W. and Taylor, P.L. (1994) Biotype Status of Hessian Fly (Diptera: Cecidomyiidae) Populations from the Eastern United States and Their Response to 14 Hessian Fly Resistance Genes. Journal of Economic Entomology, 87, 1113-1121. https://doi.org/10.1093/jee/87.4.1113

[6] Nielsen, H. (2017) Predicting Secretory Proteins with SignalP. Methods in Molecular Biology, 1611, 59-73. https://doi.org/10.1007/978-1-4939-7015-5 6

[7] Crane, Y.M., Crane, C.F., SanMiguel, P. and Schemerhorn, B.J. (2019) A Multiplexing Strategy for Mesoscale Targeted Sequencing of Populations. Academia Journal of Agricultural Research (Accepted).

[8] Robinson, J.T., Thorvaldsdottir, H., Winckler, W., Guttman, M., Lander, E.S., Getz, G. and Mesirov, J.P. (2011) Integrative Genomics Viewer. Nature Biotechnology, 29, 24-26. https://doi.org/10.1038/nbt.1754

[9] Vib-Ugent Center for Plant Systems Biology (2019) Calculate and Draw Custom Venn Diagrams. http://bioinformatics.psb.ugent.be/webtools/Venn

[10] Meta-Chart (2019) Venn Diagram Maker Online. https://www.meta-chart.com/venn

[11] Dusa, A. (2018) R Package Venn. https://CRAN.R-project.org/package=venn

[12] Schemerhorn, B.J., Crane, Y.M., Cambron, S.E., Crane, C.F. and Shukle, R.H. (2015) Use of Microsatellite and SNP Markers for Biotype Characterization in Hessian Fly. Journal of Insect Science, 15, 158. https://doi.org/10.1093/jisesa/iev138

[13] Aggarwal, R., Subramanyam, S., Zhao, C., Chen, M.-S., Harris, M.O. and Stuart, J.J. (2014) Avirulence Effector Discovery in a Plant Galling and Plant Parasitic Arthropod, the Hessian Fly (Mayetiola destructor). PLoS ONE, 9, e100958.

https://doi.org/10.1371/journal.pone.0100958

[14] Onstad, D.W. and Knolhoff, L. (2014) Arthropod Resistance to Crops. In: Onstad, D.W., Ed., Insect Resistance Management: Biology, Economics and Prediction, Academic Press, London, 2nd Edition, 293-326. https://doi.org/10.1016/B978-0-12-396955-2.00009-6

[15] Bankevich, A., Nurk, S., Antipov, D., Gurevich, A., Dvorkin, M., Kulikov, A.S., Lesin, V.M., Nikolenko, S.I., Pham, S., Prjibelski, A.D., Pyshkin, A.V., Sirotkin, A.V., Vyahhi, N., Tesler, G., Alekseyev, M.A. and Pevzner, P.A. (2012) SPAdes: A New Genome Assembly Algorithm and Its Applications to Single-Cell Sequencing. Journal of Computational Biology, 19, 455-477. https://doi.org/10.1089/cmb.2012.0021

[16] Camacho, C., Coulouris, G., Avagyan, V., Ma, N., Papadopoulos, J., Bealer, K. and Madden, T.L. (2009) BLAST+: Architecture and Applications. BMC Bioinformatics, 10, 421. https://doi.org/10.1186/1471-2105-10-421

[17] Zhao, C., Shukle, R., Navarro-Escalante, L., Chen, M., Richards, S. and Stuart, J.J. (2016) Avirulence Gene Mapping in the Hessian Fly (Mayetiola destructor) Reveals a Protein Phosphatase 2C Effector Gene Family. Journal of Insect Physiology, 84, 22-31. https://doi.org/10.1016/j.jinsphys.2015.10.001 
Supplementary Table 1 Nucleotide Sequences of the 52 Secreted Salivary Proteins in Fasta Format with Identity, Forward Primer (5'-3'), Reverse Primer (5'-3'), and Genbank Accession in Each Defline

>S1 ACAGTGTCAGGGAATCCGAC TCTTTAGGCGTTTCATTGGG EZ422040.1 ACAGTGTCAGGGAATCCGACGAGACAAGAAGCTGATGAGATAACGAAAATTTGGCAGACGAGCTTATGG AATAATCACATTCAGGCAGAAAAGTCAGTACCCTGTATTAAATATGCTATGATACTATAGCATCAAAAAC ATCATATTAAATATGTTCTAAATTTTTTGTTTCGAAAATTTTTGTTTGAGCAGATATATGATTGACGATAA TCGAGCGATATTCATGTTTAAGGATGGAAGTCAAGCATGGGAAGCAAAAGATTTTCTAATCACTCAAGAT CGTTGCGAAAGTGTATCTGTTGAGAATAAAGTCTATCCAGGAAAAGGAAGTCCCAATGAAACGCCTAAAG A

$>$ S10 AAATAATGGCCGCTCATCAC $\quad$ GGCCCAAGGAATGGATTATT $\quad$ FM984359.1 AAATAATGGCCGCTCATCACGATTCACTTTTATACAATCTTCAGCCAAACGTTTCAATGCTTGCGGACAAT CCGAGCGAACTTTTGTCAAATCTGGACGCAAAACACCACGACCAACTTTTAGCAGAATTTCATCTTTGTTA TTTATATGATTATAGGGCAATTGAAGCGTTAACAATTCATACATAACAATTCCGAATGCATAAACATCACT TTGAAATGAATATGGATTTGATTCTCGCATTCGAATTACTTCGGGTGCCATCCAAATTAAACTGCCCGTTG GATATTCCGATTGTTGTTCGCCGGACCATCGTGCTTTTGCCGTTGCAAGACCAAAATCGCCAATTTTCACG GACAAATCTTCGTGCAAGAAGATATTATACGATTTGAGATCTCGATGAATAATATTCTTTGCATGTAAATA ATCCATTCCTTGGGCC

>S103 ATCGAAATTGGTGGCGATA CCAACACACAGGCACTCACT IK139521.1 ATCGAAATTGGTTGGCGATAACGCATTCATTACCGCTAACAAAATGGTATTTTGATGAAACCAGCGAATG GACATTGGATTATCCGCCATTTTTCGCTTACTTTGAATGGACACTGTCACAGGTGGCGAAATTCTTTGATC CAAATATGCTTAATGTGAACAATTTGAATTATTCATCGTTTGAAACGATTCTTTTTCAACGTCTTTCGGTC ATTTTAACGGACATTGTTTATGCAATCGGTGTAAAAAGGTAAGTGAGTGCCTGTGTGTTGG

>S117 GCACTTTGTCCAAAAACGGT ACTGGGCAATCCATCTGAAC I EG370693.1 GCACTTTGTCCAAAAACGGTTATTCGATCGGAATCACCGCCAAAACTCACGACATTATCATTCACCCATTG AAGTGCCATTTGTTGATCTTTTAAACCCATATTACCGGAAAAATCGGATGTTCCAAGAGCTAAAAAACCA AATGCGCCCAGCCGATAATTTATCGTTACAACACAAACATCCTTTTCGATCAGAAAATCAGGACCATAAT AATAATCCATTCCAGATCCTTCGGCAAATCCACCACCGTGAATGAAAAACATCACCGCAAATCTTTCATTT GAATTGCGATGCACTGCAAATAGTAGTTGATGGGAATGAATTTCGCACAGTAGAGGTCGGCTACTGCACA TAGTATACTGTTATGTGTGCAACTTACCAGGAACGAAAATATTCAAATAAAGACAATCCTCACTTTGTTCA GATGGATTGCCCAGT

>S12 GGTTGCAGACTCCGAAAGAG CCCAAAATCCCCTATCATCC EV465518.1 GGTTGCAGACTCCGAAAGAGTTAAACCATTTCTTCCGAATGATGTCATGGAAAAAATCCTCCTTAAAACT GATTTCAAGACATTACAATCGGCCATAAGAGCTTTTGCAGTACCTCCGGATACGATCGTTATGACCCAAA TATTACAAGATCACATAATTGAAATTGTGCCACTTAAAAAGGGTACTGATATCATTGAAGTTGAATCCAA CGGCGAATTATTCCTTCAAATCACTAACTATAAACAACTTGAAAATTTCTTCAAAAAATTTGGTGACCGAG TGAAGAAAATTGCCATTGATTACAGACCATTAATTGAGGATGATCAATACAAAAAAAATTACGAATATAT TATCCAAAAATGTGCAAAAAATTTAGAAGAGCTGCGAATTGAAACTAAGGATGATAGGGGATTTTGGG 
>S122 TCATCGAGTGCTTTCATTGC CAGGCGAACGTAAAGATGGT DQ440437.1

TCATCGAGTGCTTTCATTGCTTGAGCGAAATGATGACCATGATCGATTCGTCCACCTGCGAAATAATTTGA AGTCATCAATATTCAACGATGGTTTAAATGGTTACGAAAACGATTATTACATACCTTCGACAAATAAGAA AAAGCCATTTTCATCTTTTTGTAATATTTTGATTGCGGCCTCAGTCATTTCGACGAGATTTGGTTGCTTCAT TCGGTCTGCGTCTAAGTTGAAATCCATGTGACCGGACGAAAAAAGTCCCAACACTTGATCCAATTCTTCC AAATTTAAATTATCTAATTCTTCTTTGCGTGATATAAATTTGCCTTTTGGTTTATCGCTCAACCATTCTTCA ATTAAATTACGACCATCTTTACGTTCGCCTG

>S127 CTAAATTTGTCTCGCCCAA CCCAGCCGATATTTAGGTGA DC235742.1 CTAAATTTGTCTCGCCCCAAACCAGCTACAAATGGTAAACATTGAATGTGTGATTTATTGAAGCATAAATA ATTCCTTTTGAAATAATAATAACCTGTGAATAATGTCCGGTCTTTGGTGACCAACTTGCTCCCCATGCATA TTTCCGAACTTCGTTAAACCAGTTTTGGATCCTGGATGAAAAACTTCCTTCGTGTGGTCCAAGTGGAGCTG TACTCCACATGAGCGCTAAATTTTGGCCCATTGTGAATCTATCTGAAAAATCAAAATCGACAATTTTTCCA TTAGAATATATAACTCTACTATGTCACAAGAATTTGAACGTTCGGTTCATCGAGCGCATTATTCGCGGATT ATTTGTGTGTTTCTCGAAAGGAAAATCTCACCTAAATATCGGCTGGG

>S14 TTTCCAGACCGAACAGAAG CGAGTGCGTTAAAAGCCAAT $\quad$ EV466481.1 TTTCCAGACCCGAACAGAAGTTAGAATGCTTTGTAGAAGCGTAAGTTTGAATCATTTAATTACATTAGGA ATCTACTAAAGAATTATAATTGATTCGGTTTCAGTCTCGCAGATAAGATAAAATATGGTCAGGTGCCTGA AAGAAACAATCATTTGCATACGTGGAATATGTGGGCAGGTGTACGTCAGCAATTCAAAATTGAATGTGTT GCTGACTTTGAAAGAAGGTGAGTTATGACAGAATCTCATTTAACCACACAAACAAAAAGACAATAAATTA ATATATTGAATGTATTCAGGATTTTGGAAAGATCTACAACTTTCGCAGAAAGAAACAAGCATCTATTGGG AACATTGGCTTTTAACGCACTCG

>S144 TCGCAAAAGAAAGCCAGATT CTGGCAACCCATACCAATTC CK810674.1 TCGCAAAAGAAAGCCAGATTTTAGTCGTGACATTTCACGAGTTCCGATCAGTCTTTTTTCTTCGATTTCGA CGAGCTCTGTGAGCTGATCATAAATATTTGACGCCCATTCTGGAATACTAAACATTTTGGTTAGATTTTTA TCTTTATGAAAATGTAACACAAAGCTCAAAACATGTTATTTACGTTCTATTGTGGAGCTGTTGAACCATGA GCGTATCATAAATGCAAATGATATCTCTGAAATTTTCCATTGAAAGAGCACTTTTTTCGCCAACATAATCG TAAAGCCATTCATATTTTAAACGCCAAGCACTAAATTCAGGCTTATTAAAATGTTTTCCAAAAGGAATTCA AATCGATCGCATCGTTTACCGCCAGCCAAAACATAATCCATTTTAGACGGAATAGTATGAATTGGTATGG GTTGCCAG

>S148 CTGGTGGCTTAGCTTCAAGG ATTCCGATGTTTTTGTTGCC $\quad$ FD933207.1 CTGGTGGCTTAGCTTCAAGGGCGCAACTTGATTCGGATGCATTGAAACCAAATGATTCAATGATACTGCG AGCCTTTTCTACTAATTCGTGCCTTAAGTTGGGTGGTGTGTCACGATAATGGAATGTTAATAGTGGACCTT TGTTTTCGACCCAGGCACCGTCACGGCATATCTGTTCAGGAAAACAATGCCATTTTAAATGAAAATCTTTT CAAATTGAAATCGATGAGGTTGATCAAGTACTTACCGATTCCTGTAGCGATTTAAGCAAATCGCTAACTTT ATCTTCATATTGACTGGGAAGTGGATGAACGAATTTACTACCATCCGAATGTAAAATCTCTAAGCCATGG TTACCGGCATAGGTGATACCTTCAATTCCGACCATTTTCTTAACATTTTCCACATTTCTGCCGGATATAAC GGCAACAAAAACATCGGAAT

>S15 TGAATGGATAATGGTGCGA ACCGTGACCGAGTGGATTAG $\quad$ EZ422485.1 TGAATGGATAAATGGTGCGAGTACGTATAACTATTTTTCAGCCAAAAGTCGTGTCGGACCGGTGAGAAAT 
CTAAAGTTTCATTTCATTTGGTTAGTCATTTACTGCTTAATATTATTGATCGAAAAAAATTTTTAGATTTCA AGAAAATTGGCCGAACTGTTGGATATGCTCTATGACAATGGCATGGATTTGGGGGATTTACTTATTGTAG GACATTCATTGGGGGCACACATTGCTGGGCGAGCATGCAAACAAATGAAATCTCCAGGAAAATGTGGTGT TATCATTGGTCTAGATCCCGCATCCGTTGGATTTGATTTTTTTGAAAGAGATTTGCGTTTAACTGAAACAG ATGCTGAATATGTGCAAATTATCCACACGGATGGAGATAAATTTGGTTTTTCTAATCCACTCGGTCACGGT

>S18 CTTTCTTCAGTGCGTGGACA CCGAATTGGGTTGTTATCG HS094723.1 CTTTCTTCAGTGCGTGGACACTGAAATATGAACCGTTCTAGAATAGCCCAACTCATTCATGAAATGACAA CGAAATACCTGCAAAACATCATCCTTCAGCATTTTGAATATGGCATCGCACACTACTGGAATAATTTTCGA TATTGTCGACTGCGGTATACGGAACAGATATTGCAATGACATGAAGCTGTCGCCAGTAGCCAGAAAACGC AATGTCAACGCCAAACGTTCACCTGGTGACACAGCATAGCGCATTGATGTGTCCTCTTTTTTTATGAGCGG CGACACCGAATTCAACAGATTATTAAAATCCACAATATTCATACGAAGGAAATTCTTGTATAAATACGGC ACCTCTTGTTCTAGCTCAACAAGCAATTGCGAATAACAACCCAATTCGG

>S19 GTACATCATGCAGCATTCGG TTCAGGCATGCCAAATGTAA EW780297.1 GTACATCATGCAGCATTCGGCGATGAGCCGGCAGTCATTGAGCAATTGTCGAAGGCTGGCGGTGACTTGA ATGCCCGAAACAAACGACGTCAGACAGCATTGCATATCGCTATCAATAAAGGCCATTTAAACGTTGTAAA AACATTACTCGAACTTAATTGTCACCCAAGTTTACAAGATTCAGAAGGCGATACACCACTCCATGATGCT ATATCCAAAGAACAAGATGAAATGCTTTCGCTGCTACTCGATTTTGGTGCTGATATAACGCTAACAAATA ATAATGGTTTCAATGCTTTACATCATTGCGCCCTTAAGGGAAATCCCAGTGCAATGAAGATTTTGTTATCG AAAACGAATCGGCCCTGGATTGTTGAAGAAAAAAAGATGATGGATATACAGCATTACATTTGGCATGCCT GAA

>S2 TTCACCTTGGGTTCAACGAT CCGGCAACGAAAATCTGTAT $\quad$ EZ423269.1 TTCACCTTGGGTTCAACGATTTTTGATCGAGGTCAATTAATTGTTCCGTATAGCAAAATTGCTATGTATGC GATTTGTCTCATTTTACCACTTGGATTGGGTGTATTAATTCAAAAACGCCTACCAAATTTAGCAAAAGTAC TTGTTCGCATATTGAAGTCGTTTTCAGGTGTACTCATTATTTTCATCATCGTTTTTGCCATTTTCACTAATC TTTATTTATTCAAACTGTTCACGTGGCAGGTGAGTCTAATTTTCAATGAACGTATTTTAATTGTATTGTGG ATTACACACTGATTACATATCAAATTCGATTGATTCCATGCAATTCGGAAAGTGACTGATATGACTGATAA AAATGAAAATAGAATGAAATAACCGAACACATGGTTTCTATTTTTATAACATACAGATTTTCGTTGCCGG

>S21 CGCAACATGCTTTCCATCTA CAATTGCCAGTGTTGTTTGC HS094723.1 CGCAACATGCTTTCCATCTATGGCACCTAAGCATTGAGGGAAATTCCATCGTTTATAGAAGTCGTTCGATA TATTTTTCCATTCTTCTTCTGACGATGGAAACTATTTAAATAAAACCAATAAAATTAACTAAAAATCTGAA TGGAATCGAGAATTCAATGTATACCTTGAAATGACGATCTTTCAGTGATTGATAAATTGCTTCGCACGTTT CAGGGACGATTACTGAGATGCATGGTTGAGAAATACGGAACATATATGAGAGAGATTTAAAACTTTCACC AGTTGCCAGGTATCTCAACGTTAACATTAATCGTTCGCGTGATGAAATTGCTTCACGAAGGATAGTATCTT GCCTTTCAATCAATGGACCAACGAGACCAAGAAGCAAATCAACATTGTTTTTTGACATTCGTAGAAAATT TGCAAACAACACTGGCAATTG

>S22 ATAATTGGCAAGCGGTTC CTTTTTGGTGTGTACGTCCG AY388560.1 ATAATTGGCCAAGCGGTTCCAATCAACTGCACATACATTGGAATCAACTAATTTCGCCCAATCAATAGCT GTCAAACGCATCCATCCTGCAAATTAAAAACTGTCTGATGACTAATTGTAGGAATTAAGTATGTTATCTAT 
GCATAATGCCAGGACTCCGCTTGACATCTACTGGTAGATATACATTACCGCCATCTAACCCCCCTAACCA ACCATGAATAATAAAGTTTACAGGTTTTTCTAAATTTAGTTTAGAATGGATATCTGGATCATTGAAGTAGA TTTGATGCAGTTCTTTTTTTTTGCTACAGAAAATTTAAACAAATATTCGATTCATCGTTAAAATACATAGT TATTACTTCAAAAAGTATTCGCAAGAAAATATTTCACAATGTACTTACGGACGTACACACCAAAAAG

>S25 ATGTTCGAACTCCCAACGAC AGCGTCACAAAAATTACCCG DQ440225.1 ATGTTCGAACTCCCAACGACAATGGGGTTACATCAAACAAAAGCATTCTTAGTTTGTTATCGGTCAAATC GCCATTTAAGATTGCAGCTTGTACAGCGGCACCGTTTGCGACAATTGTGTCTGGATTCGAGACCTTAAGA ATTTCAATACCATCGAAAGCATTTTTCAATAGCTCTTCAATTTTTGGAATTTTCGATGATCCTCCGATCAT AACGATTTTGTCGATCTTCAGATGTTTATCTTTAACTTTTGCGTCGTTGATTGCTTTCCGCATCAAATCGAT GGTTTTCTCGAACAATGATGCATTCATTTTTTCGAACTGCGAACGGGTAATTTTTGTGACGCT

>S27 ACATTGAGGCAATTTCGACC TACGATTGAagAatGCTGCG DQ440225.1 ACATTGAGGCAATTTCGACCGTTGGCCGAAACGTTGGTGGAAAAGATATTGATCAAATTATCGCTTCAAA TTTGATAAAAACTATGGAGGTGAATGGCAACGCCTTCGAAAAAACAAACATCAACTGCGTCAATTTGCTA AACACAAGCAAGGAGGCAAAAGAGTGTTTAACGTCCTCGTCAACGGCTTTTATCAATTTCGAATCTATTA ATGAATTAGATAATTATATTGTTTTCAAGTCGTCAATGAGCCGCCAAACGTTCGAAGAGTTGAACAAGGA TCTATTTAATTCATTTTTAGTAATAGTAGAAAAGGCCATATATGATGCAAAAATTGATAAGGCAAACATTG AAGATGTTGTCTTGGTTGGTGGTTCGGCTCATATTCCAAAGTTTCGCAGCATTCTTCAATCGTA

>S28 TGTTTGATTGCGTTTTTCCA TTCCAGAATGCTTACACGGA $\quad$ FM961982.1 TGTTTGATTGCGTTTTTCCAACTCGAACTGCGGTATGAAGCCAAAGTACCGATTGATGAAATTGAATTCAT AGAAATCAATACTCAAATCTATTCTTTTAGCGGTTTGGAGTGGCACTTGTACGTGCAGGCCAACTCAAGC TGAATCAATCACACTTCAAGAATGATCTAAGGCCCATTGATGAAACCTGTGATTGCAGTACATGCAAACA TTATTCACGGGCTTATTTGCACCATATAGTTCGAGTTGAAGCTGTAGGTGCAAGTTTGCTAACTGTTCACA ACGTTGCTTATCAGGTGAATTAATATCATTTTATTTCGTTAAATTAGTGTACTATTTACCTGAATTTTTGTT TATTCAATAGCTACGACTGATGAAAGATATCCGTGTAAGCATTCTGGAA

>S29 TCTTCGTAGCAACGCTGATG ATTCAAGAGCCCTTTGGATG EW780428.1 TCTTCGTAGCAACGCTGATGTTGGTCCAGAATCATACAATTGGGAATTTGAAACCTCAAACTCAATCAAA GCCAATGAAGCTGGACAATTGAAACAAGTGGGTGAACAGCCTGCTATCACCTCTCAAGGAGCTTTCGAAT GGGTCTCTCCCGAAGGAGAACAAATCCAAGTCAGCTATGTTGCTGACGAAAATGGATACCAACCACAAGT AAATTTATGTTCCAATCCATTCAGACTGACCAATGACATATTATGTTCGATCAATGCTCTCCAAAATCGAA GGCTGAATATTTGATTATTTTTTGTTCATTTTGTTAGGGCTCACACTTGCCAACTCCACCACCAATCCCAG AAGCCATCCAAAGGGCTCTTGAAT

>S3 CTTATCGCGGCACAATTCTT GGATCGCAAACACTTTTGGT AY752844.1

CTTATCGCGGCACAATTCTTCGCCAGCCATTTTTATAAAGGCATTAGATGGTGTAATATCATAAATACCCA GCCAGGATGATATTGTCTATAAAATATGTAAGATTAAATTATTCAACACATATATGCAACATGCAATTTTT CCGATCAACATACCCCCAACGCCGAAACGAATGGCGCGATCAAACGAATTGGTGGACTTTCAACGTGCGA CATAAACGCAACTGGAGCGAGTGCATGCATAATGTTGACCTTGGAAAGATATGCAGGTTTTTGAGAGCTC ATCACATAAAAAGCGGTGGTTCCTTGTGAATGACCGATGAACTGTAGTTGCGATACACGAGTTTGGTTCA AAATATAATCGATCGTTGCTGGCAAATCGTAAATACCAATTTGATGCCAGGAAAATGACCAAAAGTGTTT GCGATCC 
>S30 GCAAACCTGTATGCCCAACT GCGAAAGTTTTATGGGCAAA EE571743.1

GCAAACCTGTATGCCCAACTGGACCTGCTAACGGATTACATGACCAAACGACAGCAAATGAGTCGTAATC CGTATCCAAAACATGCATTGTTGTGTCATAATTCACCGGCAATCCAGTATATTTGACCGTAAATGTTGCTT CATCATTTTTAGCCGTCACATTCATAACACCCGAAACAATACGCTGAACATTTGTACTATAACAAAATGAA AGGAAAAATGATTTCAATTCAAAAGCAACAACAATAGTTTTTGGTGTACATTATTGGCAAGGAAAAAAAA ATATCTTACAATCGATTGGTCATTTCGTTATTAACATAGACTTTGCCATCTGCACGTCTTTCATATGTTACC ATAACACAACGTGATGCCAATTCAGATACAGCAAAATAGCGTTCTGCTTCGTACCATTTGCCCATAAAAC TTTCGC

>S31 ATAACCAACGCCAAATCCAG TAGGTAGAATTGGCGGAGCA AY752808.1 ATAACCAACGCCAAATCCAGATGTTGATCCTTTTTGGGCTTTAACAAAATTCGATTCAAATCCTCGTTGAA TATTTTGATAGTTGATATTTATGTATTGATCGCGTTCTTCGCGGTTTTGCTCATGTAAAAATCCGGCTGAA TGCATAAATTCGTGTAATACAGTTCCCAATAATGTTGTACAAGCAGGCGATTGTAAATTGACTGTTTGTGC TCCGCCAATTCTACCTA

>S32 ACGAGTGGCTTAAACATGGG CGCCGAACTGGTCTTTCTAC DW715640.1 ACGAGTGGCTTAAACATGGGACATGTGCTAGCGTGCTTGCCGATCTAAGTACAGAAAATAAATATTTTGG TCAGGGATTAACATGGTTTCAACAGTACGCAATGGATGTATTCCTTGCCAAAGAAAATATCGTCCCTGGA TCACAATATAATATAACAGATTTCCAATCCGCTATTAAAAAAACATTGAATATAAATCCGTCAATACATTG CATCAAAGAGAAAAGATCCGGAAATCCTTACCTGGCCGAAATTCGAATTTGTTTCAATAGAAATCTCGAG CTCGTGAACTGTGATGGCATTAAATTTTATACTGAAGAAAATTTGATCACAAATTGCCAAGACGACAAGC CCGTTTGGTATCCCAATGCAGTTCCGGAATATTTTCTAAATCGAAAGCCCGAACATGTAGAAAGACCAGT TCGGCG

>S34 TTACTCAGCACGCCATTGAC ATGGTGGGATTCCAAAACAA EZ423076.1 TTACTCAGCACGCCATTGACTCGAAACTCGTTTGTTGAATGAAATCCCGTGGTAATTTTGTTGATATTGAA GCCCGTTGTAGCAACTTGGCACCACACTTGTGCCGCTGAGATCCAAAATAATTGCTGTTGATTGTATTTAA GATCGGGTAATATATCTTCGGGTCCATTTCGTTTAACCCAATTGTTGTACGCAATATAGGCTTCTTTTGCT CCACCATTATCAGCAATATTCTCACCTGACCGGTCAAAAAAAAAATTCATTCATCAAGTGATTTAAGTAAA TGCGTAGATATGAACTTGAATCTACCTTGAGTGTTGATTCCATTTAAACTGAGATTCGTTTTAAGATCCGT GTAATTTCCATACTGTTCAATAATACATTTGGCTTTTTCCAAAAATTGTTCTCTTGTTTTGGAATCCCACCA $\mathrm{T}$

>S35 TAATTTCTGGGCCTCGTTG TATGCCCAATATGCATCGAA EV466763.1 TAATTTTCTGGGCCTCGTTGCAAAATGGTAGGTCACAGATTTCACATTCACAATATTTGACACCCAACAAT TTGAGCATGTCCATTTCCTTCTGTAATTCCTCTAATTTCTTCCTCACTTCTATCATTTCGTCTCCGGATTGG TTATCGACAACTATAATATTGGTGCTATATAGTTTTCGGATATCAGCGAACACTGCTCGAGCCTTACAAGT TGGACAGCATTGTAAGCGAACTGGGCAAACCGAAAGCCAACTGAGAGAAGAAGAACATGAATTTTATAA AGGTGTCGAACCAATCAAATACATACAACATACTTCCTTACACAGGAATCTCCAAAAATATGTCCGCACT TTAATGCAACGATGAAATGGCTACCGGACAATGATACAGGTTCGATGCATATTGGGCATA

>S36 ACGGCATGTCACAAATTTCA CACAATCGAAGCGAGTCAAA EV466763.1 ACGGCATGTCACAAATTTCACATTCGCAATACTTGACACCCAACCTCTTCAGCATATCCATTTCACGTTGT AATTTTTCAAATTTCTCGTTTAGCGTGTGCATTTCTTCTTCGGATTGGCCATCGACAATTAAAATATTGTTA 
GCATATAGCTTCCGAATTTCACTGGATACTGCACGGGCCTTACAGGTTGGACAGCTTTGTAAGCGGGATG GGCATTCTGACAGCCACCTGAGAGAAAAATATTTTGGATTTTATGATGTGTCAAACCAATCAGTTTGATA CAACATACTTCCTTAAGCAGGAATCACCAAAGAGATGCCCACACTTTGTTGAGACGAGGAAATGGCTACC AGACGATGTACAGGCCTCGTAACAGATTGGACATTTTAATACTTCTAAATCGTCAGTATCTGGTTTGACTC GCTTCGATTGTG

\section{$>$ S37 CACCGAATTTCGACCAAGAT TGCAGATTCGCAGGTATGAG EV465518.1}

CACCGAATTTCGACCAAGATTCATCAATCAATGATATCAACGAAGATCTGCCATTTTTGCCAAATGATTTG ATTGAAATGATTATCAGTAAAACTGATTTCAAAACAATACATGAGGCCGTACAATCACGGATACTACGTA CAACTGACATCGTAGTGAATAGAATTTTACAAGATCACATAATTATAATTGATAGTCGTAAACGCTTTGAT GTTACTGATATCAATGAATTTCATGCACAAGGCGACTCATACCTGCGAATCTGCA

>S39 GCGGAGAATTTGATGAagGA TTTCCACTCTTCAACGGAC HS094723.1 GCGGAGAATTTGATGAAGGAATTGGATATTGAAATGCCGGCTCTGTATACGAATTTTGTGCGTTTATCAA AAGAGAATTTTGAAGTGCTTCTGGGGTTTGTTGGGCCAATGATTGCTCGGAGCGATACTGTTCTTCGTGA GGCGATTTGTGCCCGGGAAAGATTAATGATTACGATGCGATATTTGGCGACAGGTGAAACATTCAAATCG TTATCATACATGTTTCGTGTGTCTCAGCCGAGCATATCGTCAATTGTGCCGGAAACATGTGAGGCTATTTA TAAAGCCTTGAAAGATCGCCACATGAAGGTAAATTGAATTCATTTGTTCGGTGTGTGATTTGTTTATTTGA TTAGTTTTTGTGTATCGTGTGTCGTTTTAGTTTCCGTCGTCCGTTGAAGAGTGGGAAA

>S4 ATTTGCGCACATAATGCAAG CGCAAACTTCGTACTCGTCA $\quad$ FD933570.1 ATTTGCGCACATAATGCAAGTAGTCACGACGGATGACAATTGTACGTTGCATCTTCAATTTTTGGACAATA CCGTTCAAGATACGACCACGGATACGTACATTTCCGGTGAATGGGCATTTGTTGTCGATGTATGTACCGT CTATTGCCTATTTAATTTAAAAATAAAGAAAGAATTGAATGAGTTACTTTTTTTCTCTCGTCACGGCTGAT AAGGACTTGAGTATGGTTTACTTCTTTTGGTGTTTTGAAGCCCAAACCAACATCTCGGTGGTGACGAGTAC GAAGTTTGCG

>S40 GTTAACAATGCTACGCGCAC GATGCATTGCACCATAAACG AY552055.1 GTTAACAATGCTACGCGCACTTTGTCTAAAAGTTGGAATACAGATTCTATTACGTGAATACAATTTTGAAT CGAAAAACAAACCAACATTCAATGAAGATGACATTGTAAATGTATTCCCAATCGTTAAGCATATAAATCC ACGTGCCACCGATGCTTACAACTTCTATACAACCGGCCAAACAAAAATCCAACAAGGTCATTTCAAAGAA GGATATGAATTGATAAGTGAATCGTTGAATTTATTGAACAACGTTTATGGTGCAATGCATC

>S42 TCGAGTCCATTGGGAAGATT AACGGATTCACGAGTCTTGG I CF541909.1 TCGAGTCCATTGGGAAGATTCATTGATCTTATGTTGGTCCCATATGGAAAGGCTAGTGTAAGTGATCAAC AAAAAAAATATCAAATGGATTATTTTCTCATATTCATAATCGATTTATTTGCAATAAAATAGTTTCAAACC CAAGGATCGGATGTTGTATTCGAATGTCAACATGGACCAAATGAATGTTATGGTAATAAAGTGCATGCTT GTGCTATTGAACATATTCAAGTAAGTTTAACTGAATCTTTGTAGTTTGATTTAATTTTAACATCGTTTCAA GAGATTATTAACTATTATTATTATTATTGCAGGTGAATTCTTATCAAAATACCAAGACTCGTGAATCCGTT

>S44 CAATGTCCGGTAAGCCAGTT TCGGAACAATTTCAAGGACC EZ422768.1 CAATGTCCGGTAAGCCAGTTTTATCACATTTGACATAATATTCGTTGGTATTTTTATTTAAGACGGCACCG ATATGACGATTTATTTCCTTAATTTCCTCACGTGGTCCAACTATTATACTTGTTCCAGTATCCAGGATCGC TTCACAACCTCGATTACATCCCGTAAATAATAAATTATTTTGATCGGAGCTTTCACCACGCTGAAATATTT 
CAATCCGAGACATACGAAATTGCCAATAACCCAATTTCGTGACGGGCAGCCAAAATTCTGGCTTAACATC ACATCCGCCAATTATCAATTCACCACCAATTTCAGCCGTTTGATTATTCATATGAAAGCAAAAAATCTTTT CATTGATTACACCTTGCTCTTTGAGTCGATCAAGTGCTGTATAATTCCAGGAGGTCCTTGAAATTGTTCCG A

>S46 AGCTGACGACTCCCAAATGT GCTTTGGACAAAATCCGCTA EE264606.1 AGCTGACGACTCCCAAATGTATTGCTCATCATCGTTGTGTTTCGATGTAACGGTGACCTTATCGGCAACCA AATAGGCTAAGTAGAAACCAACACCGAATTGTCCAATCTTACTGATGTCAGCACCAGCTTGCAATGCCTC CATGGATGCCTTTGTTTCGGATTTAGCTATTGTACCAAGATTGTTGACCAAATCGGCTTTAGTCATACCAA TACCAGTATCAATGATGGTAAGAGTACCGGCGGCTGTGTTTGGAATGATTTTGATGTACAATTCCTTGCC GCTGTCGAGTTTTGATGGACCAGTGAGTGATTCATAGCGGATTTTGTCCAAAGC

>S48 TCCAAACCGAAGTAAATCGC ATCGGCATTATGTTTGGTGG EE264606.1 TCCAAACCGAAGTAAATCGCATGATGAAATTAATCATCAATTCGTTGTATCGTAACAAAGAAATTTTCCTT CGTGAATTGATCTCGAATGCATCGGATGCTTTGGATAAGATTCGTCTGTTGTCATTGACAAATCGAACGG AAATTGAAACACATCCAGAGATGAAAATTCAAATTAGAGCCGACAAAGATAACAAGGTGTTGCACATTAT CGATACTGGTATTGGTATGACAAGAGAGGATTTGAGCAACAATTTGGGAACCATTGCTAAGTCCGGAACA GCCGATTTTTTGGCAAAACTGCAAGACAGTCCAAATGAAAATGAAAATGATATGATTGGTCAATTTGGTG TTGGATTTTATTCGGCATTTTTGGTTGCCGATCGGGTTGTTGTCACCACCAAACATAATGCCGAT

>S5 AAGGTGAGTTTGTCAGGCG AGAGCCCAAAACGACAGAGA EV465518.1 AAGGTGAGTTTTGTCAGGCGTGGCATATCAAATGAAAGATTTCCAATTCCAAATAATTCGTCCATTTGGTC TTCATCCATTTCGTCTTCATCAAACTCAATATAAAAGTGGGTCACTGATGGAAGTTTATATACTGGGTTAT TGTTTTTTCGTAACATAAAGTTTTCACCAGGATTTTCAACTTCAAGTGTTTCTAACTTCGTCAAATATTTTG CAATCGACTGCATTGTCTCTGTCGTTTTGGGCTCT

>S51 CGCCGAATCTCAGGATTAAG AGACTCATCCAACCGGACAC EV465518.1 CGCCGAATCTCAGGATTAAGTCGCCATGTCGATTATATTCATGGATTCCACCATTGAACTCATTTGGTTTA ATAAGAATTATGTAATCTTGTAATATTCTGCTCATTACAAGGTTGGTTGGAGGTGTTATTCCCCATCTTAC GGTCTGTTTTATTGTTTTGTAATCAGTTTTACTAACAATCAATTCAATTAAATCATTCGGTAAAATTAGTTT TTCTTCTTTAATATCAATGGTTGGTGCATCTTGTGTCCGGTTGGATGAGTCT

>S59 CTGGTGCACGATACCAAAGA CAATGCTTTTCTGCCATCAA FM965180.1 CTGGTGCACGATACCAAAGAGTGACGATTTCATGCGTGTATACTCGAACCGGAATTCCAAACGCACGTCC TAATCCAAAGTCAGCGATCTATTTCAAATAAAAATGTAATAAATATAAATAAAATGTAATAAATAAATGT TCTTTTGAATGTTTGGCTTGTAAAAAAATTATGGATTGAACCTTAATAACTCCTTCCCGATTGATCAATAA ATTTTGTGGCTTCAAATCACGATGTAAAATTCGTCGTTGATGGCAGAAAAGCATTG

>S6 ACAATGAAAAGGCTGAAC GCCCATCATTTGGCTAAGAA DQ440398.1 ACAATGAAAACGGCTGGAACATGCCTTCGTGTCTCTTTTTGATAGTACCACTGTTATGGGTATGTTCAACA AGTCCACCGGATGCACGAAGTCTCGATTCGATATCAACAAGCAAATTGTACCATTCGGTCGTACCTTCAA AATGATTATAAAACTTTGGATTTGGTGCACCAAGCAAATCGAGTAGCACCAACATGTCCTACGAACATAA AAAAAAAAATGAATTTATCAATGAAGCACCCGAAGGAAATTGAACTGCTGAAATGAAAATTGATTTACAA TATGTTTCAATTTGCCACTTGCATGCCATTTCTTAGCCAAATGATGGGC 
>S62 AATCAAACATGGCCGGATTA CGCTGCCAGAGAAGAATACC CK810674.1

AATCAAACATGGCCGGATTATTTCCTCCCAAAGATGATCAGATATGGAATAAGAATCTAATGTGGCAAGC GATTCCAATACACACAATACCAGAATATATGGACTATATATTGGCTGCGAAAAGATCGTGCGCCCAATAT GAGCATGCTCTTAAGAGGTATCAACAATCGCCTGAATTTAAAGTTCTATTGGACAAATTTAGGCCCTTGAT GTTTTATTTGGAGCAGAGTACCGGAAAAGCCATTAGAACACTAACAGATGTAGGAAATCTATACAATACC CTATGGATTGAAAATCTCAAGAACAAAACGTAAATATGTCACATGAAATATATATCGAGTGAGTCTGAGT TTATTCTCTATTTTCTTAGTTTGCCAGAGTGGACAAAAAAGGTATTCTTCTCTGGCAGCG

>S65 CTTCGGTTGAATGTGTCCCT EE265141.1

CTTCGGTTGAATGTGTCCCTTTTTTTATGTAAATATCTAATTTCAATTTATGTGGAATATTTCGTTCGATTT TTATCCGTAAACAGAGTCCGATTAGCGTTGCCAATGAGCAATGGGGTACGGTTGGATTGAATTCCACGCG AATCTACAATTGATCATCAAAGAATTAATTTAAAATCATGGTGAATTTCTCCTCTGAACCTGCAAATGAAC ATGTTTGCTGACAAATCTAATTCAAACGCATATTTTTGCTTACCACCTGAACATCATCACTTGTGGGTGCC TGCACGAATATTCCATCTTCATAAACCACTTTCAAATCTTCCAATGTGGATGGCTTTTCAGGATCCCGTAT

>S67 GGCTGATGAATCCCAAATGT TTCTTCGGTTTGCATAGGCT $\quad$ EE264606.1 GGCTGATGAATCCCAAATGTATTGTTCATCATCATTGTGTTTCGCTGTTACGGTAACTTTGTCGGCAACCA AGAAGGCTGAGTGAAAACCAACATCGAATTGATCAATCATATCAATATCAGCCCCCGCTTTCAATGTTTC CATAAATGCAATTGTTCTTGATTTAGCAACTGTACCAAGTTTGATGATCATATCGGTTTTAGTCATACCAA TACCAGTATCAATTATGGTAAGTGTACCAGCAGCTTTGTTGGGAATGATTTTAATGTACAATTCTTTGCCG CTGTCGAGTTTTGATGGATCAAAGAGTGATTCATATCGGATTTTGTCCAAAGCCTATGCAAACCGAAGAA

>S7 AATGATTCGGCCATCAAATC GACAAGGGCCCACAATCTTA EV465518.1

AATGATTCGGCCATCAAATCGTTCTCCACTAATGGTGAGCTTTTTCAGGTTTGGCATATCAAATGAAAGTC TTCCGATTGCAAATACATCGTCCATTCTACTACCTAAACTAATATCAAAGTGTATCACTGATGGAAGCTTA TATACTTCATTATTGTTTGCTCGTAACAAAACGTTTTCACCAGGAATTCCAATTACAAGTGTTTCTAACTT TGTTAAATATTTTGCAATCGATTGCATTGTATCCGTCCTGTTTGGCAATGCGAGAGAAATTGTTGTCATGT TCCTATTATTTGCGAAAATATCATTAAGATTGTGGGCCCTTGTC

$>$ S71 CGTGCATGACCAACAATACC GAAGGTATGCCGATGTTGG EH114134.1 CGTGCATGACCAACAATACCTGGATATCCGGCACACGTTCGATTACCAAAATCTTGATAAATTCCAAATTT CAAACCGTTCGAATGAATCTGTGGAAAATTTATTATGGCAATAAAATAATAAGAGCAATAATGATCAAAA CAATTTCCCAATGTTCATTCAAATACTAACGTGTTGACTTAATGATTTCATGCCACGTGGAAAACGTTGTC GATCGGCAACCATTTCACCTTTTGATCCACGTGATTTTTCCAACCAACAATCATCAACGTTCACATATTCA TATCCAACATCGGCATAACCTTC

>S72 TTGCAATGGACGAATTTGAA AACCGGTCACTTTTTGCATC $\quad$ AY190633.1 TTGCAATGGACGAATTTGAAAACCATTTCATATGGTGAATGTTTACGGGAATTTCCATTTTTGATATTTAG AAAAAGTGTTATTTGTGCTCGGGGCGAACAAAAGGAATCAGCTTGCCATGGTGATTCCGGTGGTCCACTT ATCGATAGCAAAACAAATACTTTGATTGGTTTGGCTAGTTTTGTTTCACATAGTATGTGCATTGTGCCTTC ATTTAAATAGCAGGATACAAAAATCGCTGTGCATTGTGAGTGAGACCTTTAATTATAATTGCCATTTTTGA TTACAGAAGGTTGTCATCTTGGTCTTCCGCAAGGCTATACTTATGTTCAATCATATTTTCCATGGATGCAA AAAGTGACCGGTT 
>S73 CATTTCGCCATTCGAAAACT TGGCTAAGACCACCATAGGC U11052.1 CATTTCGCCATTCGAAAACTAATAATTTTATTGTTTCATTTTCGTTCACAGAATGGTATTCGGTAAGCATT ATCACGGTTACTCAAGTTCAATTCGTCCAGGTGTCTTCAATGAAATTGCAACTAGCGCGTTGCCAGCATTT ATGACCATGTTGCCAAATGGAATGAACAATGAATCAACAACAATTGAAACATTATTAACGACTTCGGCTT TGTTAAAAACCTTAAAACCAAACAATTTGGATTTCAATGAATACTGGACACCAATCGCTCTGGCTATTCAT CGTAGCCGTGACCATGGCATTCCAGCCTATCACAAAGCATTGAATCTTTGTGAAGAACGCTTACCAAATA CAAATGGACAAAGACTCACATTTGCCGATCTTCAAGCCTATGGTGGTCTTAGCCA

>S74 AAAGAATGTGCCGTCGAAAC TTCATCTTTGTTTGCACCGA EV466783.1 AAAGAATGTGCCGTCGAAACATCATTTACACCAGAAGATCTGACCAAGCTTCGCACCAATCCAAAAGCTG CCGTTGGCGATGCTAAAGCACAAGTAAGCTATTCGCTCATTTGCAGAAATTCAATTTTGAAAGGAACCAC CAAATTGTAGACCTTTCATTTGAAATTTGAAACATTGATTTTTTTTTTGGTTTCACATTTCAACAGAAATTC AGTAAATGTTTCCTCCACAAATTGGGCTTCATCAATGATGCCGGTGATTTCCAAGAACATGTTGTCATCGA AAAATTGTCAAAAGGTGGTGACCGTGCCAAAATTGAAGAAATCGTTAAACAATGCAAATCCGTCATCGGT GCAAACAAAGATGAA

>S75 CGGAGCTTGGAAAAGTGTC TCCCATTGTCTTTGTGCGTA $\quad$ FD933933.1 CGGAGCTTGGAAAAAGTGTCATAATTTCGCCATTTTCAGTGGCAACCTCTCTCACACTTCTGTTACAAGCA ACAAATGGGACAACTTGCACACAGTTGAAAAATTGTTTGCATTTGCAGGACAATAATATTGCGAAATCAC ATGTGGCCAATCAGTTTCGCAATTATTTTGCATTAATAAAAAAGAGTGCTGGACAATCGGAACTGATGAT TGTCAATCAAATCTATGTACAACAAACATTTCGATTGAATAAAGAGTTTCATGAAATTGCAGCCAATGAA TTTGATGCCGGTGTTGAATCCGTGAATTTTTCCAACACAAACGAAACAACACAATTAATCAATAATTTCAT ACGCACAAAGACAATGGGA

>S78 ACGAGTGGTTGCCAATTTTT TCACTCAATCGCAGTGAACC EU934306.1

ACGAGTGGTTGCCAATTTTTTTGGGCGTTTCGAATATGGAAAAAAATCGTTTAATTTATCGCATTGAGCCG GGCAGCTATGTTAATGATTATGATCAAACAATTGATCCATCTGTTCTAAAGTCACATGCAACAGCCGCATT CAGATATTTTCATTCACAAATTGAAGGACGCCTCGAGTAAATATTTTGTTTGAATGAGATTAAATTATAGA ACTCACTTTCTTGTTCTTTATTTCTCTTTCATTTTTCCTCATTTCTCCATCTGCATATAGTCTTATATCAGAG ATTCGATCGAGCACTGGTTCACTGCGATTGAGTGA

>S8 CTTTTCAGGCACTTGGTCC CAAAATCTGCGCATTTGCTA EV466345.1 CTTTTTCAGGCACTTGGTCCACTTGTTGTTGATGCTGGGGATCGGGAAGATGGAGGTTCAAGATCTGGGA GGCGTTTGGACAGAAAGCATTCACTTGATAAAATGAAAAAAGTTGTTAGGACTTTGGCAGAAAAGGCAGA AGTGTGTTGGTTCCGTCCACAAGTTCTCGGAGACTCTGAAAAATCTTACAACGACGGTCCTGCGCAGGAT TACAGAGGAGATGTTTATGTCACTAATTACAATAATAAGAAACACTGGCAGAAGAGAGCAGAAATAGCA AAAAAAAATGGTGATCAGGCAGCGTTGGAACTAGCAAATGCGCAGATTTTG

$>$ S88 CGACACGCCTTTTAGTGTCA CGATTAGGCGAATGGAACAT $\quad$ DQ440124.1 CGACACGCCTTTTAGTGTCACCTGGAGTTTAATTTTGCTTCCCAAGGCTAAAAAAAACAACAAAAATACC TAAATAAATTGAAAATAACTCACCAATAGCCTATTAACCATTCGGTAATGAAGATGTGTGTCCCCATCCTG ACACAACCATCGGAACATCATCAAAAGATTTTTGCTTCCATTTAATATCGGCTGGCAAACATATTGGTTTA ATCCATTGCGTATTTGGTACACTGTAAGCCAATCGGAGTAGTGCAATATCATTTTCATGATTCAGTGTTTC TTCTTGATAATTTTCATGAATGAATATCTCGTTTATTGGAACATCCAAGACTGGATCTGAGCATACATTAT TTTGGCAATCAATCTCTTCGCTAATGTTCCATTCGCCTAATCG 
>S9 GAAGCAAACGAACGGAACTC AGGTGAGCATTCGCAGTTCT $\quad$ EV465518.1

GAAGCAAACGAACGGAACTCAAATTGAATAAAATATTTCCCAAACTTGAATGTTTGAGGCTGTCTGGAAC CATAGCCGATATAAATTGCTTATCAAATGTAAAAAGATTGAAGAATCTAACGCTGGGCTACGGCTCGACC CACTCAATACAAGAAGACCAATTTGATCATATTTTTCAAAATAATACTGGACTGACAATGATTTCTCTCTA TGGGCCTAATACGAGGAAGACAATGCAGTCAATTGCCGACAATTTGAAAGAGTTAAAAACACTTGAAGTG AAAAAACCTGGTAGAAAGTTTTTGTACCGAGCAATCAATAATCCAGTGTGTGAACTTCCAAAAGTGGAAC ACTTTGAAATTTTATTTCATGATATGAACGAAGTATTCGGAATCGGAAATCCTTCATTTCATATGCCAGAA CTGCGAATGCTCACCT 